Article

\title{
Bound Electron Transitions under the Influence of Electromagnetic Wave in Constant Magnetic Field
}

\author{
Vladimir Zhukovsky \\ Faculty of Physics, Moscow State University, 119991 Moscow, Russia; zhukovsk@phys.msu.ru
}

Received: 16 July 2020; Accepted: 15 August 2020; Published: 17 August 2020

\begin{abstract}
Motion and radiative transitions of an electron in a magnetic field under the influence of an external electromagnetic wave are studied for various confining conditions in semiconductor, graphene, in quantum wells, and relativistic generalization in terms of the Klein-Gordon equation are considered. In particular, the following problems are discussed. The so-called cyclotron resonance, which may appear in graphene, is studied with indication for appearance of the so-called frequency-halving. The problem is solved for two-dimensional massless charged particle, whose gapless nature is protected by sublattice symmetry. The exact classical calculation of this effect is undertaken in the framework of a $2 \mathrm{D}$ classical equation for a zero-mass electron. We also find an exact solution of the Schrödinger equation for charge carriers in semiconductors under the influence of an external magnetic field and in the field of electromagnetic wave with an account for their radiative transitions. Solutions of the relativistic Klein-Gordon equation in this configuration of electromagnetic fields are found as a certain generalization of the results obtained for the non-relativistic case. These results may serve as a first step for further efforts to find exact solutions of wave equations for quasiparticles in solid state structures in external fields.
\end{abstract}

Keywords: synchrotron radiation; cyclotron resonance; frequency-halving; semiconductor; graphene; sublattice symmetry; quantum well

\section{Introduction}

Synchrotron radiation (SR) is a well-known phenomenon. Its theory developed already in the middle of the last century is in perfect agreement with experiment [1-3]. The very well-known properties of SR have found a great deal of theoretical and technical applications both in studying optical properties of materials and in high-energy physics, as well as in astrophysics, where superstrong magnetic fields of the order of the Schwinger value $H_{S c h}=4.41 \times 10^{13} G$ are expected $[4,5]$ to exist. An outstanding phenomenon called "Sokolov-Ternov effect" [6] have found numerous applications in the high-energy physics. Just for an example, we mention here the relation between Unruh and Sokolov-Ternov effects, studied by E.T. Akhmedov and D. Singleton [7]. Another example of an experimental use and verification of the theoretical study of SR and related topics, we mention an experiment of the VEPP-2000 [8] to measure the cross-sections of hadron production in $e^{+} e^{-}$collision that clearly indicates an interference of scattered radiation caused by the bend of electron trajectory, which demonstrated very good correspondence with the theoretical calculations [9].

Cyclotron radiation contrary to synchrotron radiation is emitted by non-relativistic charged particles. Its emission in graphene and a possibility of Landau-level laser was studied in several papers (see, e.g., [10]). This physical situation is related to the so-called cyclotron resonance (CR), see, e.g., [11-13], which may appear in graphene, the planar monoatomic layer of carbon [14].

The higher intensities of laser radiation and of the constant magnetic and electric fields, as well as the increased interest in the physics of narrow-band semiconductors, call for further development of the theory. The influence of a strong alternating electric field on crystals with narrow forbidden band of 
width comparable with intraband motion energy in external fields was first considered by Keldysh [15]. The advent of powerful optical-radiation sources has stimulated experimental and theoretical investigations of undulators and free-electron lasers (see, e.g., [16], where high-harmonic x-ray undulator radiation for nanoscale-wavelength free-electron lasers was investigated, and Refs. $[17,18]$ ), as well as of semiconductors in strong electromagnetic fields.

These investigations are carried out in many cases in the presence of an external constant magnetic field that plays a major role in the study of the electronic states in semiconductors. A 2D graphene model, where the gapless nature is protected by sublattice symmetry, was studied in various papers with particular forms of magnetic field. As some of the numerous examples we mention paper [19], where generation of electric and vacuum currents was demonstrated in a superposition of a homogeneous magnetic field and an Aharonov-Bohmvortex in graphene, and also [20], where, in particular, chiral symmetry breaking due to appearance of condensates and phase transitions under external conditions like temperature, chemical potential and Aharonov-Bohm magnetic fields were studied.

Recent advances in semiconductor technology considerably extended possibilities of microelectronic devices. Theoretical study of the optical absorption in a quantum well with the semi-parabolicOscillations of the optical absorption coefficient in crossed electric and magnetic fields potential plus the semi-inverse squared potential (SPSIS) was carried out in [21]. The higher intensities of laser radiation and of the constant magnetic field, as well as the increased interest in the physics of narrow-band semiconductors, call for further development of the theory.

The general aim of this paper is to find most common interesting features of excitations and radiative transitions of charge carriers in external fields in low-dimensional quantum systems, such as semiconductors or graphene. The exact classical calculation of cyclotron resonance in graphene with indication for appearance of the so-called frequency-halving is presented in Section 2.

The behavior of charge carriers in semiconductors under the influence of an external magnetic field and in the field of electromagnetic wave is of long-lasting interest. We present in this paper an exact solution of the Schrödinger equation for such a system (Section 3) with an account for its radiative transitions (see Section 4). Further generalization of the method of Section 3 are made in Section 5, where we used results obtained for the solution of the non-relativistic Schrödinger equation to construct some wave functions as solutions of the relativistic scalar wave equation-Klein-Gordon equation. These solutions may be of interest as a first step for further efforts to find exact solutions of wave equations for quasiparticles in semiconductors in external fields.

Semiconductor crystals with wide forbidden bands (E, large compared with the intraband motion energy) are the subject of papers [21-24], where a quantum well with the SPSIS potential was considered in the presence of a static magnetic field. The influence of a constant magnetic field was also taken into account in Refs. $[25,26]$. In this paper, we included in our calculations, moreover SPSIS potential and a static magnetic field, an electromagnetic wave field. We followed the method developed in Section 3, where the magnetic field and radiation field were taken into account exactly. Then, due to inclusion of the confining potential, the optical absorption coefficients become strongly affected by cumulative influence of both a static magnetic field and the confinement frequency and the intensity of incident electromagnetic wave. These factors are exactly taken into account in our solution (Section 6).

\section{Cyclotron Resonance}

The energy of electrons and holes in graphene are described by the following linear equation with zero effective mass:

$$
\mathcal{E}_{ \pm}(\mathbf{P})= \pm v_{f} P= \pm v_{f}|\mathbf{P}|,
$$

where $\mathbf{P}=\left(P_{x}, P_{y}\right)$ is the $2 \mathrm{D}$ electron momentum, and $v_{f} \approx 10^{8} \mathrm{~cm} / \mathrm{s}$ is the Fermi velocity. 
A free charged massive particle (e.g., electron), with charge $e=-e_{0}<0$ and mass $m$, placed in a magnetic field $\mathbf{B}$, moves in a circular orbit in the plane perpendicular to the field $\mathbf{B}$ with the cyclotron frequency (for non-relativistic velocities, $v \ll c$, with $c=3 \times 10^{10} \mathrm{~cm} / \mathrm{s}$ )

$$
\omega_{c}=e_{0} B / m c .
$$

If the frequency of an applied electromagnetic wave $\omega$ is getting near the cyclotron frequency, $\omega \simeq \omega_{c}$, the cyclotron resonance [11-13] takes place. It is quite useful in solid state physics for studies of metals and semiconductors. It is interesting to note that as it will be seen in what follows, the CR line in graphene turns out to be rather broad, which is characteristic for relativistic or massless particles.

\subsection{Equations of Motion}

The equation of motion for a massless quasi-particle with the linear dispersion relation (1) in a constant magnetic field $\mathbf{B}=(0,0, B)$ and under the influence of an applied electromagnetic wave $\mathbf{E}(t)$ reads

$$
\frac{d \mathbf{P}}{d t}={ }_{-}^{e} \mathbf{v} \times \mathbf{B}+e \mathbf{E}(t), \quad \mathbf{v}=v_{f} \frac{\mathbf{P}}{P} .
$$

One should note that the electromagnetic wave propagates along the $z$-direction, and hence the electric field and magnetic field of the wave are both in the $X-Y$ plane, where, as we assumed, an electron is confined. Since the Fermi velocity $v_{f}$ is about $1 / 300$ that of the velocity of light $c$, the magnetic field of the wave $\mathbf{H}_{0}(t)$ can be neglected.

How can one relate this equation to the Hamilton method? Equation (3) should follow from the Hamiltonian function. For $m \neq 0$ we have

$$
L=-m c^{2} \sqrt{1-v^{2} / c^{2}}+e / c(\mathbf{v A})-\mathbf{e} \mathbf{A}_{0} .
$$

Variation gives the Lagrange equation of motion

$$
\frac{d}{d t} \frac{m \mathbf{v}}{\sqrt{1-v^{2} / c^{2}}}=\frac{e}{c} \mathbf{v} \times \mathbf{B}+e \mathbf{E} .
$$

For $m=0$, we have ambiguity with $m \rightarrow 0$ and $v \rightarrow c$.

Now via Hamiltonian equations. We have

$$
H=v_{f} \sqrt{(\mathbf{p}-e / c \mathbf{A})^{2}}+e A_{0}, \mathbf{p}-e / c \mathbf{A}=\mathbf{P} .
$$

Hamiltonian equations read

$$
\begin{aligned}
& \frac{\partial H}{\partial \mathbf{p}}=v_{f} \frac{\mathbf{p}-e / c \mathbf{A}}{\sqrt{(\mathbf{p}-e / c \mathbf{A})^{2}}}=\mathbf{v} \\
& \frac{\partial H}{\partial \mathbf{r}}=-\frac{d \mathbf{p}}{d t}=-\frac{d \mathbf{P}}{d t}-e / c \frac{d \mathbf{A}}{d t}
\end{aligned}
$$

where

$$
\frac{\partial H}{\partial \mathbf{r}}=\frac{\partial(\mathbf{v}, \mathbf{p}-e / c \mathbf{A})}{\partial \mathbf{r}}+e \frac{\partial A_{0}}{\partial \mathbf{r}},
$$

and

$$
\frac{d \mathbf{A}}{d t}=\frac{\partial \mathbf{A}}{\partial t}+\left(\mathbf{v} \frac{\partial \mathbf{A}}{\partial \mathbf{r}}\right) .
$$


Here

$$
\begin{aligned}
& \frac{\partial(\mathbf{v}, \mathbf{p}-e / c \mathbf{A})}{\partial \mathbf{r}}=-e / c \frac{\partial(\mathbf{v}, \mathbf{A})}{\partial \mathbf{r}} \\
& =-(e / c)\left(\mathbf{v} \frac{\partial \mathbf{A}}{\partial \mathbf{r}}\right)-(e / c) \mathbf{v} \times\left(\frac{\partial}{\partial \mathbf{r}} \times \mathbf{A}\right),
\end{aligned}
$$

so that

$$
\begin{aligned}
\frac{d \mathbf{P}}{d t} & =-e / c \frac{d \mathbf{A}}{d t}+e / c\left(\mathbf{v} \frac{\partial \mathbf{A}}{\partial \mathbf{r}}\right)-e \frac{\partial A_{0}}{\partial \mathbf{r}}+(e / c) \mathbf{v} \times\left(\frac{\partial}{\partial \mathbf{r}} \times \mathbf{A}\right) \\
& =(e / c) \mathbf{v} \times\left(\frac{\partial}{\partial \mathbf{r}} \times \mathbf{A}\right)-(e / c) \frac{\partial \mathbf{A}}{\partial t}-e \frac{\partial A_{0}}{\partial \mathbf{r}} \\
& =e \mathbf{E}+(e / c) \mathbf{v} \times \mathbf{B}
\end{aligned}
$$

which is just Equation (3). Without the electromagnetic field, $\mathbf{E}(t)=0$, the energy of the particle is conserved $\mathcal{E}=v_{f} P=$ const, and the particle rotates in the magnetic field with the frequency

$$
\omega_{B}=e_{0} B v_{f} / P c=e_{0} B v_{f}^{2} / c \mathcal{E}
$$

that depends on its energy $\mathcal{E}=v_{f} P$.

\subsection{Circular Polarization}

To study the cyclotron resonance of the massless particle with the dispersion law (1), we assume that the radiation frequency $\omega$ coincides with the electron rotation frequency $\omega_{B}$. Moreover, we propose that the wave is circularly polarized in the direction coinciding with the sense of the particle rotation

$$
\mathbf{E}_{0}(t)=E_{0}(\cos \omega t, \sin \omega t) .
$$

Thus, we have the equation of motion

$$
\frac{d \mathbf{P}}{d t}=-\frac{e_{0}}{c} \mathbf{v} \times \mathbf{B}-e_{0} \mathbf{E}_{0}(t), \quad \mathbf{v}=v_{f} \frac{\mathbf{P}}{P} .
$$

First consider the case without an electric field. Then

$$
\mathbf{P} \frac{d \mathbf{P}}{d t}=-\frac{e_{0}}{c}(\mathbf{P}, \mathbf{v} \times \mathbf{B})=0,
$$

and $\mathbf{P}^{2}=$ const, $\mathcal{E}_{ \pm}(\mathbf{P})= \pm \mathcal{E}, \mathcal{E}=v_{f}|\mathbf{P}|=v_{f} P=$ const.

We have $\mathbf{P}=\left(P_{x}, P_{y}\right)=P(\cos \varphi$, $\sin \varphi)$, then

$$
\begin{aligned}
& P \dot{\varphi}(-\sin \varphi, \cos \varphi, 0)=-\frac{e_{0} B}{c} v_{f}(\cos \varphi, \sin \varphi, 0) \times(0,0,1) \\
& =\frac{e_{0} B v_{f}}{c}(-\sin \varphi, \cos \varphi, 0),
\end{aligned}
$$

so that $\dot{\varphi}=\frac{e_{0} B v_{f}}{c P}$ and

$$
\mathbf{P}=\left(P_{x}, P_{y}\right)=P\left(\cos \left(\omega_{B} t+\phi_{0}\right), \sin \left(\omega_{B} t+\phi_{0}\right)\right) .
$$

Let us emphasize that contrary to the non-relativistic case, rotation frequency of the massless particle $\omega_{B}(13)$ depends on its energy. 
Now let us include electric field into consideration

$$
\begin{aligned}
& \frac{d \mathbf{P}}{d t}=-\frac{e_{0}}{c} \mathbf{v} \times \mathbf{B}-e_{0} \mathbf{E}_{0}(t), \quad \mathbf{v}=v_{f} \frac{\mathbf{P}}{P}, \\
& \mathbf{E}_{0}(t)=E_{0}(\cos \omega t, \sin \omega t) .
\end{aligned}
$$

Now instead of (16) we have

$$
\mathbf{P} \frac{d \mathbf{P}}{d t}=-e_{0}\left(\mathbf{P}, \mathbf{E}_{0}\right)=-e_{0} P E_{0} \cos (\varphi-\omega t) .
$$

Next, we have

$$
\begin{aligned}
& \mathbf{E}_{0} \frac{d \mathbf{P}}{d t}=-\frac{e_{0}}{c}\left(\mathbf{E}_{0}, \mathbf{v} \times \mathbf{B}\right)-e_{0} E_{0}^{2} \\
& =-\frac{e_{0}}{c}\left(\mathbf{B}, \mathbf{E}_{0} \times \mathbf{v}\right)-e_{0} E_{0}^{2} \\
& =-\frac{e_{0}}{c} B E_{0} v_{f} \sin (\varphi-\omega t)-e_{0} E_{0}^{2} .
\end{aligned}
$$

Equation (19) can be rewritten as

$$
\frac{d P}{d t}=-e_{0} E_{0} \cos (\varphi-\omega t)
$$

While Equation (20) is transformed as follows:

$$
\begin{aligned}
& \frac{d P}{d t} \cos (\varphi-\omega t)-P \frac{d \varphi}{d t} \sin (\varphi-\omega t)= \\
& =-\frac{e_{0}}{c} B v_{f} \sin (\varphi-\omega t)-e_{0} E_{0}
\end{aligned}
$$

and recall here Equation (21). It should be noted that Equations (21) and (22) to within a change of notations coincide with corresponding Equations (4) and (5) from [11]. In fact, trivial transformations give for Equation (22)

$$
P \frac{d \varphi}{d t}=\frac{e_{0}}{c} B v_{f}+e_{0} E_{0} \sin (\varphi-\omega t)
$$

Now let $E_{0} \rightarrow 0$ in Equations (21) and (23). Then

$$
\begin{gathered}
\frac{d P}{d t}=-e_{0} E_{0} \cos (\varphi-\omega t) \rightarrow 0 \\
P \frac{d \varphi}{d t}=\frac{e_{0}}{c} B v_{f}+e_{0} E_{0} \sin (\varphi-\omega t) \rightarrow \frac{e_{0}}{c} B v_{f} .
\end{gathered}
$$

To simplify solution and according to initial statement of the problem, let us assume resonance condition $\omega=\omega_{B}$, and hence

$$
P=\mathrm{const}=\hat{P}, \frac{d \varphi}{d t}=\mathrm{const}=\frac{e_{0} B v_{f}}{c \hat{P}}=\omega_{B}=\omega .
$$

Now let us introduce deviations $\hat{P}_{\mathcal{E}}$ and $\phi$ from these constant solutions

$$
P=\hat{P}(1+\varepsilon), \varphi=\hat{\varphi}+\phi, \text { where } \hat{\varphi}=\omega_{c} t+\varphi_{0} .
$$


Let us start with Equation (21). We have

$$
\hat{P} \dot{\varepsilon}=-e_{0} E_{0} \cos \left(\phi+\varphi_{0}\right),
$$

and

$$
\begin{gathered}
P \frac{d \varphi}{d t}=\frac{e_{0}}{c} B v_{f}+e_{0} E_{0} \sin (\varphi-\omega t), \\
\hat{P}(1+\varepsilon) \frac{d \varphi}{d t}=\frac{e_{0}}{c} B v_{f}+e_{0} E_{0} \sin (\varphi-\omega t), \\
\hat{P}(1+\varepsilon)\left(\omega+\frac{d \phi}{d t}\right)=\frac{e_{0}}{c} B v_{f}+e_{0} E_{0} \sin \left(\phi+\varphi_{0}\right), \\
\frac{d \phi}{d t}+\varepsilon \omega+\varepsilon \frac{d \phi}{d t}=\frac{e_{0} E_{0} \sin \left(\phi+\varphi_{0}\right)}{\hat{P}}, \\
\frac{d \phi}{d t}(1+\varepsilon)+\varepsilon \omega=\frac{e_{0} E_{0} \sin \left(\phi+\varphi_{0}\right)}{\hat{P}},
\end{gathered}
$$

We introduce a dimensionless parameter

$$
\xi=\frac{e_{0} E_{0}}{\omega \hat{P}^{\prime}}
$$

so that Equations (28) and (31) take their final form

$$
\begin{gathered}
\frac{d \phi}{d t}(1+\varepsilon)+\varepsilon \omega=\omega \xi \sin \left(\phi+\varphi_{0}\right), \\
\dot{\varepsilon}=-\omega \xi \cos \left(\phi+\varphi_{0}\right) .
\end{gathered}
$$

Now let us study the case of a weak field

$$
\xi=\frac{e_{0} E_{0}}{\omega \hat{P}} \ll 1
$$

with $\varepsilon \sim \sqrt{\xi} \ll 1$. Then Equations (32) and (33) take linearized form

$$
\begin{gathered}
\frac{d \phi}{d t}=-\varepsilon \omega+\omega \xi \sin \left(\phi+\varphi_{0}\right), \\
\dot{\varepsilon}=-\omega \xi \cos \left(\phi+\varphi_{0}\right) .
\end{gathered}
$$

Let $\varphi_{0}=\pi / 2$, then we have

$$
\begin{gathered}
\frac{d \phi}{d t}=-\omega \varepsilon+\omega \xi \cos \phi \\
\dot{\varepsilon}=\omega \xi \sin \phi,
\end{gathered}
$$

or

$$
\frac{d \phi}{d \tau}=-\frac{\varepsilon}{\sqrt{\xi}}+\sqrt{\xi} \cos \phi, \frac{d \varepsilon}{d \tau}=\sqrt{\xi} \sin \phi,
$$

where $\tau=\sqrt{\xi} \omega t$. Since $\varepsilon \sim \sqrt{\xi} \ll 1$, and $\frac{\varepsilon}{\sqrt{\xi}} \sim 1$ we have

$$
\frac{d \phi}{d \tau}=-\frac{\varepsilon}{\sqrt{\xi}}, \frac{d \varepsilon}{d \tau}=\sqrt{\xi} \sin \phi,
$$


then

$$
\frac{d^{2} \phi}{d \tau^{2}}=-\sin \phi
$$

which is the equation for nonlinear oscillations. Its solution can be linearized, $\phi \ll 1$, and then the solution for $\varepsilon$ is found from the second equation in (39).

\subsection{Linear Polarization}

Let us assume that the external electric field $\mathbf{E}_{0}(t)=E_{0}(\cos \omega t, 0)$ is linearly polarized in the direction along the $x$-axis. Now instead of (16) we have

$$
\mathbf{P} \frac{d \mathbf{P}}{d t}=-e_{0}\left(\mathbf{P}, \mathbf{E}_{0}\right)=-e_{0} P E_{0} \cos \varphi \cos \omega t .
$$

Next, we have

$$
\begin{aligned}
& \mathbf{E}_{0} \frac{d \mathbf{P}}{d t}=-\frac{e_{0}}{c}\left(\mathbf{E}_{0}, \mathbf{v} \times \mathbf{B}\right)-e_{0} E_{0}^{2}= \\
& -\frac{e_{0}}{c}\left(\mathbf{B}, \mathbf{E}_{0} \times \mathbf{v}\right)-e_{0} E_{0}^{2}= \\
& =-\frac{e_{0}}{c} B E_{0} v_{f} \sin \varphi \cos \omega t-e_{0} E_{0}^{2}
\end{aligned}
$$

Equation (41) can be rewritten as

$$
\frac{d P}{d t}=-e_{0} E_{0} \cos \varphi \cos \omega t
$$

Now we transform Equation (42)

$$
\begin{aligned}
& \mathbf{E}_{0} \frac{d \mathbf{P}}{d t}= \\
& =E_{0} \cos \omega t \cos \varphi \frac{d P}{d t}-\frac{d \varphi}{d t} P E_{0} \cos \omega t \sin \varphi= \\
& -\frac{e_{0}}{c} B E_{0} v_{f} \sin \varphi \cos \omega t-e_{0} E_{0}^{2}
\end{aligned}
$$

or simplifying

$$
\begin{aligned}
& \left(\frac{d P}{d t}\right)^{2}+\frac{d \varphi}{d t} P e_{0} E_{0} \cos \omega t \sin \varphi= \\
& \frac{e_{0}^{2}}{c} B E_{0} v_{f} \sin \varphi \cos \omega t+e_{0}^{2} E_{0}^{2}
\end{aligned}
$$

Now let us study the case of a weak field

$$
\xi=\frac{e_{0} E_{0}}{\omega \hat{P}} \ll 1 .
$$

Then, with the use of variables $\varepsilon$ and $\phi$, introduced via equalities $P=\hat{P}(1+\varepsilon), \varphi=\hat{\varphi}+$ $\phi$, where $\hat{\varphi}=\omega_{c} t+\varphi_{0}$, we obtain

$$
\begin{aligned}
& \hat{P}^{2}\left(\frac{d \varepsilon}{d t}\right)^{2}+\left(\frac{d \phi}{d t}+\omega\right) P e_{0} E_{0} \cos \omega t \sin \left(\phi+\omega t+\varphi_{0}\right)= \\
& \frac{e_{0}^{2}}{c} B E_{0} v_{f} \sin \left(\phi+\omega t+\varphi_{0}\right) \cos \omega t+e_{0}^{2} E_{0}^{2} .
\end{aligned}
$$


Dividing by $(\omega \hat{P})^{2}$

$$
\begin{aligned}
& \left(\frac{d \varepsilon}{\omega d t}\right)^{2}+\left(\frac{d \phi}{\omega d t}+1\right)(1+\varepsilon) \xi \cos \omega t \sin \left(\phi+\omega t+\varphi_{0}\right)= \\
& \xi \frac{e_{0}}{c \omega \hat{P}} B v_{f} \sin \left(\phi+\omega t+\varphi_{0}\right) \cos \omega t+\xi^{2} .
\end{aligned}
$$

Since $\omega=\omega_{B}=\frac{e_{0} B v_{f}}{c \hat{P}}$, we find

$$
\begin{aligned}
& \left(\frac{d \varepsilon}{\omega d t}\right)^{2}+\left(\frac{d \phi}{\omega d t}+1\right)(1+\varepsilon) \xi \cos \omega t \sin \left(\phi+\omega t+\varphi_{0}\right)= \\
& \xi \sin \left(\phi+\omega t+\varphi_{0}\right) \cos \omega t+\xi^{2} .
\end{aligned}
$$

and since $\sqrt{\xi} \omega t=\tau$, we arrive, dividing by $\xi$, at

$$
\begin{aligned}
& \left(\frac{d \varepsilon}{d \tau}\right)^{2}+\left(\frac{\sqrt{\xi} d \phi}{d \tau}+1\right) \times \\
& (1+\varepsilon) \cos (\tau / \sqrt{\xi}) \sin \left(\phi+\tau / \sqrt{\xi}+\varphi_{0} / \sqrt{\xi}\right)= \\
& \sin \left(\phi+\tau / \sqrt{\xi}+\varphi_{0} / \sqrt{\xi}\right) \cos (\tau / \sqrt{\xi})+\xi
\end{aligned}
$$

Now, since $\varepsilon \sim \sqrt{\xi}$, we leave in the last equation only the following terms $\sim \sqrt{\xi}$

$$
\left(\frac{\sqrt{\zeta} d \phi}{d \tau}+\varepsilon\right)=0
$$

Now let us turn to Equation (43) for $P$

$$
\begin{aligned}
& \frac{d \epsilon}{d \tau}=-\frac{e_{0} E_{0}}{\hat{P} \omega \sqrt{\xi}} \cos \varphi \cos (\tau / \sqrt{\xi})= \\
& \sqrt{\xi} \sin (\phi+\tau / \sqrt{\xi}) \cos (\tau / \sqrt{\bar{\zeta}}),
\end{aligned}
$$

where we put $\varphi_{0}=\pi / 2$. Now we deal with equations

$$
\begin{aligned}
& \frac{d \phi}{d \tau}=-\frac{\varepsilon}{\sqrt{\xi}}, \\
& \frac{d \epsilon}{d \tau}=\sqrt{\xi} \sin (\phi+\tau / \sqrt{\xi}) \cos (\tau / \sqrt{\xi})= \\
& \frac{1}{2} \sqrt{\xi}\left[\sin \left(\phi+2 \frac{\tau}{\sqrt{\xi}}\right)+\sin \phi\right] .
\end{aligned}
$$

We put Equation (52) together

$$
\frac{d^{2} \phi}{d \tau^{2}}=-\frac{1}{2}\left[\sin \left(\phi+2 \frac{\tau}{\sqrt{\xi}}\right)+\sin \phi\right] .
$$

One can see that for small $\phi$ (large $t$ ) one has $\phi \sim \xi$ and then

$$
\frac{d^{2} \phi}{d \tau^{2}}=-\frac{1}{2} \sin \left(2 \frac{\tau}{\sqrt{\xi}}\right)
$$

and

$$
\frac{d \phi}{d \tau}=\frac{1}{4} \sqrt{\xi} \cos \left(2 \frac{\tau}{\sqrt{\xi}}\right), \frac{d \phi}{d \tau}=-\frac{\varepsilon}{\sqrt{\xi}}
$$


or

$$
\varepsilon=-\sqrt{\xi} \frac{d \phi}{d \tau}=-\frac{1}{4} \xi \cos \left(2 \frac{\tau}{\sqrt{\xi}}\right)=-\frac{1}{4} \xi \cos (2 \omega t),
$$

that is the frequency is doubled (which should be compared with the exceptional frequency-halving solution (64)).

In the opposite case of small $t \rightarrow 0$ we have

$$
\frac{d^{2} \phi}{d \tau^{2}}=-\sin \phi
$$

which is just Equation (40) for circular polarization.

\subsection{Particular Solution}

Now we study a particular solution of our equations. From Equation (32) together with Equation (33) we find

$$
\begin{aligned}
& \frac{d \varepsilon}{d \phi}=-\frac{d t}{d \phi} \omega \xi \cos \left(\phi+\varphi_{0}\right) \\
& \frac{d t}{d \phi}=\frac{1+\varepsilon}{\omega \xi \sin \left(\phi+\varphi_{0}\right)-\varepsilon \omega^{\prime}}
\end{aligned}
$$

so that

$$
\frac{d \varepsilon}{d \phi}=-\frac{(1+\varepsilon) \xi \cos \left(\phi+\varphi_{0}\right)}{\xi \sin \left(\phi+\varphi_{0}\right)-\varepsilon}
$$

Let $\xi \sin \left(\phi+\varphi_{0}\right)=y$, then

$$
\frac{d \varepsilon}{d y}=\frac{1+\varepsilon}{\varepsilon-y}
$$

or

$$
\frac{d(\varepsilon-y)}{d y}+1=\frac{1+\varepsilon-y+y}{\varepsilon-y}
$$

then putting $\varepsilon-y=x$,

$$
\frac{d x}{d y}=\frac{1+y}{x}
$$

i.e.,

$$
\begin{gathered}
x^{2} / 2=y^{2} / 2+y+C, \\
\left(\varepsilon-\xi \sin \left(\phi+\varphi_{0}\right)\right)^{2}=\left(\xi \sin \left(\phi+\varphi_{0}\right)\right)^{2}+2 \xi \sin \left(\phi+\varphi_{0}\right)+2 C,
\end{gathered}
$$

where $C=$ const is a constant of motion. Thus, we have a solution

$$
\begin{aligned}
& \varepsilon=\xi \sin \left(\phi+\varphi_{0}\right) \pm \\
& \sqrt{\left(\xi \sin \left(\phi+\varphi_{0}\right)\right)^{2}+2 \xi \sin \left(\phi+\varphi_{0}\right)+2 C}
\end{aligned}
$$

Let us choose $C=1 / 2$ (let us note that this is a sort of an "instanton" solution (see, e.g., [27])), then

$$
\varepsilon=\xi \sin \left(\phi+\varphi_{0}\right) \pm\left(\xi \sin \left(\phi+\varphi_{0}\right)+1\right)=\left\{\begin{array}{l}
2 \xi \sin \left(\phi+\varphi_{0}\right)+1 \\
-1
\end{array}\right.
$$


If we choose " -" sign (take $\varepsilon=-1$ ), then according to above equations, we shall have ambiguity in Equation (57), $d t / d \phi=0$, and $P=0$. With the other choice we shall have in Equation (57)

$$
\begin{aligned}
& \frac{d t}{d \phi}=\frac{2\left(\xi \sin \left(\phi+\varphi_{0}\right)+1\right)}{\omega\left(\xi \sin \left(\phi+\varphi_{0}\right)-2 \xi \sin \left(\phi+\varphi_{0}\right)-1\right)}=-\frac{2}{\omega^{\prime}} \\
& \text { that is } \dot{\phi}=-\omega / 2
\end{aligned}
$$

Finally, we have the solutions

$$
\begin{aligned}
& \phi=-\frac{\omega}{2} t \\
& \varepsilon=2 \xi \sin \left(\varphi_{0}-\frac{\omega t}{2}\right)+1,
\end{aligned}
$$

and in Equations (27)

$$
\begin{aligned}
& P=\hat{P}(1+\varepsilon)=2 \hat{P}\left(1+\xi \sin \left(\varphi_{0}-\frac{\omega t}{2}\right)\right) \\
& \varphi=\omega t+\varphi_{0}-\frac{\omega}{2} t=\frac{\omega t}{2}+\varphi_{0}, \omega=\Omega
\end{aligned}
$$

To simplify calculations, let us change notations

$$
2 \hat{P}=P_{0}, \omega / 2=\tilde{\omega}, 2 \hat{P} \xi=2 \hat{P} \frac{e_{0} E_{0}}{\omega \hat{P}}=2 \frac{e_{0} E_{0}}{\omega}=\frac{e_{0} E_{0}}{\tilde{\omega}},
$$

then

$$
\begin{aligned}
& P=\hat{P}(1+\varepsilon)=P_{0}-\frac{e_{0} E_{0}}{\tilde{\omega}} \sin \left(\tilde{\omega} t-\varphi_{0}\right) \\
& \varphi=\tilde{\omega} t+\varphi_{0}, \omega=\Omega=2 \tilde{\omega} .
\end{aligned}
$$

For components of $P$ we now have

$$
\begin{aligned}
& P_{x}=P \cos \varphi=P_{0} \cos \left(\tilde{\omega} t+\varphi_{0}\right)- \\
& -\frac{e_{0} E_{0}}{\tilde{\omega}} \sin \left(\tilde{\omega} t-\varphi_{0}\right) \cos \left(\tilde{\omega} t+\varphi_{0}\right)= \\
& =P_{0} \cos \left(\tilde{\omega} t+\varphi_{0}\right)-\frac{e_{0} E_{0}}{2 \tilde{\omega}}\left(\sin 2 \tilde{\omega} t-\sin 2 \varphi_{0}\right), \\
& P_{y}=P \sin \varphi=P_{0} \sin \left(\tilde{\omega} t+\varphi_{0}\right)- \\
& -\frac{e_{0} E_{0}}{\tilde{\omega}} \sin \left(\tilde{\omega} t-\varphi_{0}\right) \sin \left(\tilde{\omega} t+\varphi_{0}\right)= \\
& =P_{0} \sin \left(\tilde{\omega} t+\varphi_{0}\right)+\frac{e_{0} E_{0}}{2 \tilde{\omega}}\left(\cos 2 \tilde{\omega} t-\cos 2 \varphi_{0}\right) .
\end{aligned}
$$

We can now rewrite the result in the form

$$
\begin{aligned}
& P_{x}=P_{0} \cos \left(\frac{\omega t}{2}+\varphi_{0}\right)-\frac{e_{0} E_{0}}{\omega}\left(\sin \omega t-\sin 2 \varphi_{0}\right), \\
& P_{y}=P_{0} \sin \left(\frac{\omega t}{2}+\varphi_{0}\right)+\frac{e_{0} E_{0}}{\omega}\left(\cos \omega t-\cos 2 \varphi_{0}\right),
\end{aligned}
$$

where $\omega=2 \tilde{\omega}=\Omega=e_{0} B v_{f} / \hat{P}_{c}=2 e_{0} B v_{f} / P_{0} c$. 
As is seen, the above solution describes the motion that is a superposition of "free" motion in a magnetic field with "cyclotron" frequency

$$
\tilde{\omega}=\frac{1}{2} \omega=e_{0} B v_{f} / P_{0} c
$$

and forced motion with doubled frequency $\omega=2 e_{0} B v_{f} / P_{0} c$ (quasiresonance condition). When the wave field is switched off, $E_{0}=0$, the momentum $P_{0}$ is a transversal momentum of an electron in a magnetic field $P_{0}=\left.\sqrt{P_{x}^{2}+P_{y}^{2}}\right|_{E_{0}=0}$.

We note however that our exact solution (67) and (68) is different from approximated solutions of papers [11,12] (they, by the way, were obtained numerically and demonstrated in a graphical form). The principle difference is that we obtained the so-called frequency-halving or period-doubling (quasiresonance) (for an example, see [28,29]) of the cyclotron frequency in a magnetic field $\omega_{B}$ with zero electromagnetic wave field $E_{0}=0$, as compared to the frequency $\omega$

$$
\omega_{B}=e_{0} B v_{f} / P_{0} c=\omega / 2
$$

Thus, we can say that the exact solution obtained in this section under the condition of "quasiresonance" (69), i.e., with frequency-halving, differs from the "resonance" condition of the previous Section

$$
\Omega=e_{0} B v_{f} / P c=\omega
$$

We emphasize that in that case one was able to consider analytically only the case of a weak field.

\section{Non-Relativistic Electron in a Magnetic Field and in a Plane Wave}

In this Section we consider an exact solution of the Schrödinger equation in the electromagnetic field that is a superposition of a homogeneous magnetic field $\mathbf{B}=$ const, directed along the $z$ axis $(\mathbf{B} \| O z)$, and an electromagnetic wave circularly polarized in the $x O y$ plane. The vector potentials of the fields, neglecting the space dispersion of $\mathbf{A}(t)$ thanks to expected low frequency of the wave, are as follows

$$
\mathbf{A}(B)=(0, x B, 0), \mathbf{A}(t)=b(\cos \omega t, \sin \omega t, 0) .
$$

Assume that $\hbar=c=1$ in what follows due to the appropriate choice of system of units.

For the Schrödinger equation for the electron wave function $\Phi$ in a magnetic field and in an electromagnetic wave in our particular case (71) we have

$$
i \partial_{t} \Phi=\frac{-\partial_{z}^{2}+P_{x}^{2}+P_{y}^{2}}{2 m} \Phi
$$

where

$$
\begin{aligned}
& P_{x}=-i \partial_{x}+\beta \cos \omega t, \\
& P_{y}=-i \partial_{y}+e_{0} x B+\beta \sin \omega t .
\end{aligned}
$$

Here $\beta=e_{0} b$ and $e=-e_{0}<0$ is the electron charge, and in the framework of the effective mass approximation, to simplify notations, we will denote the electron effective mass by $m$ instead of $m^{*}$. The $y$ - and $z$-moment components are conserved $-i \partial_{y} \rightarrow p_{y}=$ const, $-i \partial_{z} \rightarrow p_{z}=$ const. Thus we have 


$$
\begin{aligned}
i \partial_{t} \Phi & =\frac{1}{2 m}\left[p_{z}^{2}-\left(\partial_{x}+i \beta \cos \omega t\right)^{2}\right] \Phi \\
& +\frac{\left[p_{y}+e_{0} B x+\beta \sin \omega t\right]^{2}}{2 m} \Phi .
\end{aligned}
$$

Let

$$
\Phi=\exp \left(\frac{-i p_{z}^{2}}{2 m} t\right) \mathrm{e}^{i p_{y} y} \mathrm{e}^{i p_{z} z} \bar{\Phi}(t, x)
$$

then

$$
\begin{aligned}
i \partial_{t} \bar{\Phi} & =\frac{1}{2 m}\left[-\left(\partial_{x}+i \beta \cos \omega t\right)^{2}\right] \bar{\Phi} \\
& +\frac{\left[p_{y}+e_{0} B x+\beta \sin \omega t\right]^{2}}{2 m} \bar{\Phi}
\end{aligned}
$$

or in the form of the Schrödinger equation

$$
i \partial_{t} \bar{\Phi}=\hat{H} \bar{\Phi},
$$

with the Hamiltonian

$$
\begin{aligned}
\hat{H} & =\frac{1}{2 m}\left(\hat{p}_{x}+\beta \cos \omega t\right)^{2} \\
& +\frac{m \omega_{c}^{2}}{2}\left(x-x_{0}+\frac{\beta}{e_{0} B} \sin \omega t\right)^{2}
\end{aligned}
$$

where $x_{0}=-\frac{p_{y}}{e_{0} B}, \omega_{c}=\frac{e_{0} B}{m c}$. Now we go over to new dimensionless variables

$$
x-x_{0} \rightarrow \bar{x}=\sqrt{e_{0} B}\left(x-x_{0}\right), \hat{p}_{x} \rightarrow \hat{p}=\frac{\hat{p}_{x}}{\sqrt{e_{0} B}},
$$

and we arrive at this form of the Hamiltonian

$$
\hat{H}=\frac{\omega_{c}}{2}\left[(\hat{p}+\eta \cos \omega t)^{2}+(\bar{x}+\eta \sin \omega t)^{2}\right],
$$

where $\eta=\beta / \sqrt{e_{0} B}$. We may now introduce the operators

$$
\hat{a}=\frac{1}{\sqrt{2}}(\hat{\bar{p}}-i \bar{x}), \quad \hat{a}^{+}=\frac{1}{\sqrt{2}}(\hat{\bar{p}}+i \bar{x})
$$

with the standard commutation relation

$$
\left[\hat{a}, \hat{a}^{+}\right]=1
$$

Then

$$
\begin{aligned}
& \hat{H}=\frac{\omega_{c}}{2}\left(\frac{\hat{a}+\hat{a}^{+}}{\sqrt{2}}+\eta \cos \omega t\right)^{2} \\
& +\frac{\omega_{c}}{2}\left(i \frac{\hat{a}-\hat{a}^{+}}{\sqrt{2}}+\eta \sin \omega t\right)^{2} .
\end{aligned}
$$


After transformation and with notation $\alpha=\frac{\omega_{c}}{\sqrt{2}} \eta \mathrm{e}^{i \omega t}$ we arrive at the following form of $\hat{H}$

$$
\hat{H}=\omega_{c}\left(\hat{a}^{+} \hat{a}+\frac{1}{2}\right)+\frac{|\alpha|^{2}}{\omega_{c}}+\alpha a+\alpha^{*} a^{+}
$$

Define the operator

$$
\mathrm{D}_{\mu}=\exp \left(\mu \hat{a}^{+}-\mu^{*} \hat{a}\right)
$$

with the property

$$
\mathrm{D}_{\mu}^{-1} \hat{a} \mathrm{D}_{\mu}=\hat{a}+\mu, \mathrm{D}_{\mu}^{-1} \hat{a}^{+} \mathrm{D}_{\mu}=\hat{a}^{+}+\mu^{*}
$$

Define the function

$$
\chi=\mathrm{D}_{\mu}^{-1} \bar{\Phi} \exp \left(i \int_{0}^{t} \gamma(t) d t\right)
$$

where $\gamma(t)=\frac{1}{2}\left(\mu \alpha+\mu^{*} \alpha^{*}\right)$.

An equation for $\chi$ can be found as follows

$$
\begin{aligned}
i \partial_{t} \chi & =i \partial_{t}\left[\mathrm{D}_{\mu}^{-1} \bar{\Phi} \exp \left(i \int_{0}^{t} \gamma(t) d t\right)\right] \\
& =\left(i \partial_{t} \mathrm{D}_{\mu}^{-1}\right) \bar{\Phi} \exp \left(i \int_{0}^{t} \gamma(t) d t\right) \\
& +\mathrm{D}_{\mu}^{-1}\left(i \partial_{t} \bar{\Phi}\right) \exp \left(i \int_{0}^{t} \gamma(t) d t\right) \\
& +\left(-\gamma \mathrm{D}_{\mu}^{-1}\right) \bar{\Phi} \exp \left(i \int_{0}^{t} \gamma(t) d t\right) .
\end{aligned}
$$

With the use of the identity

$$
\frac{\partial \mathrm{e}^{v}}{\partial t}=\left(\frac{\partial v}{\partial t}+\frac{1}{2}\left[v, \frac{\partial v}{\partial t}\right]\right) \mathrm{e}^{v}
$$

under the condition that $v$ and $\partial v / \partial t$ commute with $\left[v, \frac{\partial v}{\partial t}\right]$, with $\mathrm{D}_{\mu}^{-1}=\mathrm{e}^{-\mu \hat{a}^{+}+\mu^{*} \hat{a}}$, we have in Equation (86)

$$
\partial_{t} \mathrm{D}_{\mu}^{-1}=\left[-\dot{\mu} a^{+}+\dot{\mu}^{*} a+\frac{1}{2}\left(\mu \dot{\mu}^{*}-\mu^{*} \dot{\mu}\right)\right] \mathrm{D}_{\mu}^{-1} .
$$

Then

$$
\begin{aligned}
& \mathrm{D}_{\mu}^{-1} i \partial_{t} \bar{\Phi}=\mathrm{D}_{\mu}^{-1} \hat{H} \mathrm{D}_{\mu} \mathrm{D}_{\mu}^{-1} \bar{\Phi} \\
& \mathrm{D}_{\mu}^{-1} \hat{H} \mathrm{D}_{\mu}=\mathrm{D}_{\mu}^{-1}\left(\omega_{c}\left(\hat{a}^{+} \hat{a}+\frac{1}{2}\right)+\frac{|\alpha|^{2}}{\omega_{c}}+\alpha a+\alpha^{*} a^{+}\right) \mathrm{D}_{\mu} \\
& =\omega_{c}\left(\hat{a}^{+} \hat{a}+\frac{1}{2}\right)+\omega_{c} \mu^{*} \mu+\frac{|\alpha|^{2}}{\omega_{c}} \\
& +\omega_{c} \hat{a}^{+} \mu+\omega_{c} \hat{a} \mu^{*}+\alpha \hat{a}+\alpha^{*} \hat{a}^{+}+\alpha \mu+\alpha^{*} \mu^{*}
\end{aligned}
$$

Now we come to the equation for $\chi$ 


$$
\begin{aligned}
i \partial_{t} \chi & =i \partial_{t}\left[\mathrm{D}_{\mu}^{-1} \bar{\Phi} \exp \left(i \int_{0}^{t} \gamma(t) d t\right)\right] \\
& =i\left[-\dot{\mu} \hat{a}^{+}+\dot{\mu}^{*} \hat{a}+\frac{1}{2}\left(\mu \dot{\mu}^{*}-\mu^{*} \dot{\mu}\right)\right] \chi \\
& +\omega_{c}\left(\hat{a}^{+} \hat{a}+\frac{1}{2}\right) \chi \\
& +\left[\omega_{c} \hat{a}^{+} \mu+\omega_{c} \hat{a} \mu^{*}+\omega_{c} \mu^{*} \mu+\alpha \mu+\alpha^{*} \mu^{*}\right] \chi \\
& +\left[\frac{|\alpha|^{2}}{\omega_{c}}+\alpha \hat{a}+\alpha^{*} \hat{a}^{+}\right] \chi \\
& -\frac{1}{2}\left(\mu \alpha+\mu^{*} \alpha^{*}\right) \chi .
\end{aligned}
$$

Let $\mu$ satisfy the equation

$$
\dot{\mu}+i \omega_{c} \mu=-i \alpha^{*}, \text { where } \alpha^{*}=|\alpha| \mathrm{e}^{-i \omega t}
$$

Its solution apart from the solution of the homogeneous equation can be found

$$
\mu(t)=\frac{|\alpha|}{\omega-\omega_{c}} \mathrm{e}^{-i \omega t} .
$$

It is easy to show, using Equation (91) that the terms proportional to $\hat{a}$ and $\hat{a}^{+}$, as well as terms proportional to $\mu$ and $\mu^{*}$ cancel between themselves in the above equation and we arrive at the final equation for $\chi$

$$
i \partial_{t} \chi=\omega_{\mathcal{c}}\left(\hat{a}^{+} \hat{a}+\frac{1}{2}\right) \chi
$$

This is the standard equation for an oscillator with the solution

$$
\begin{aligned}
\chi & =\exp \left(-i \varepsilon_{n} t\right) u_{n}(\bar{x}), \\
\varepsilon_{n} & =\omega_{c}(n+1 / 2), \quad n=0,1,2, \ldots
\end{aligned}
$$

and the wave function of our problem $\Phi$ is defined through Equations (75) and (85), and is finally expressed in terms of the wave function $u_{n}(\bar{x})$ of an electron in a magnetic field $[2,30]$ with the help of the operator $\Lambda$

$$
\Lambda=\exp \left[-\frac{i}{\hbar} \int_{0}^{t} \gamma\left(t^{\prime}\right) d t^{\prime}+\mu \hat{a}^{+}-\mu^{*} \hat{a}\right]
$$

so that the final solution takes the form

$$
\begin{aligned}
& \Phi_{p_{y}, p_{z}, n}=\mathrm{e}^{i p_{y} y} \mathrm{e}^{i p_{z} z} \mathrm{e}^{-i \varepsilon_{n, p_{z}} t} \Lambda u_{n}(\bar{x}) \\
& =\mathrm{e}^{i p_{y} y} \mathrm{e}^{i p_{z} z} \mathrm{e}^{-i \varepsilon_{n, p_{z}} t} \mathrm{D}_{\mu} u_{n}(\bar{x}) \exp \left(-i \int_{0}^{t} \gamma(t) d t\right),
\end{aligned}
$$

where

$$
u_{n}(\bar{x})=\frac{\left(e_{0} B\right)^{1 / 4} \mathrm{e}^{-\bar{x}^{2} / 2}}{\sqrt{\sqrt{\pi} 2^{n} n !}} H_{n}(\bar{x}) .
$$

and the energy spectrum

$$
\varepsilon_{n, p_{z}}=\omega_{c}(n+1 / 2)+\frac{p_{z}^{2}}{2 m}
$$

Here $H_{n}(\bar{x})$ is a Hermite polynomial. 
This result for an exact solution of the equation for a non-relativistic electron in a magnetic field and an electromagnetic wave was for the first time published in [31] in 1967 with an application to radiative transitions in a semiconductor. This solution was once again reproduced by other authors [32] later in 1982.

\section{Electron Radiative Transitions}

\subsection{Intraband Transitions}

If the electron is at a level $n$ in the absence of the electromagnetic wave at $t=0$, then a strong alternating field with photon energy $\hbar \omega<\epsilon_{g}\left(\epsilon_{g}\right.$ is the width of the forbidden zone in a semiconductor) makes possible transitions at $t>0$ to the level $l$ inside the band with the following amplitude

$$
\begin{aligned}
& A_{n \rightarrow l}=<l|\Lambda| n>=\int d x u_{l}^{*} \Lambda u_{n} \\
& =\int d x \frac{(e B)^{1 / 2} \mathrm{e}^{-\bar{x}^{2} / 2}}{\sqrt{\sqrt{\pi} 2^{l} l !}} H_{l}(\bar{x}) \Lambda \frac{\mathrm{e}^{-\bar{x}^{2} / 2}}{\sqrt{\sqrt{\pi} 2^{n} n !}} H_{n}(\bar{x}) \\
& =\frac{1}{\sqrt{\pi 2^{l+n} l ! n !}} \int d \bar{x} \mathrm{e}^{-\bar{x}^{2} / 2} H_{l}(\bar{x}) \Lambda H_{n}(\bar{x}) \mathrm{e}^{-\bar{x}^{2} / 2} \\
& =\frac{\exp \left[-\frac{i}{\hbar} \int_{0}^{t} \gamma\left(t^{\prime}\right) d t^{\prime}\right]}{\sqrt{\pi 2^{l+n} l ! n !}} \int d \bar{x} \mathrm{e}^{-\bar{x}^{2} / 2} H_{l}(\bar{x}) \mathrm{D}-H_{n}(\bar{x}) \mathrm{e}^{-\bar{x}^{2} / 2} .
\end{aligned}
$$

We have

$$
\begin{aligned}
& \mathrm{D}_{\mu} f(\bar{x})=\exp \left(\mu \hat{a}^{+}-\mu^{*} \hat{a}\right) f(\bar{x}) \\
& =\exp \left[\mu \frac{1}{\sqrt{2}}(\bar{p}+i \bar{x})-\mu^{*} \frac{1}{\sqrt{2}}(\bar{p}-i \bar{x})\right] f(\bar{x}) \\
& \left.=\exp \frac{1}{\sqrt{2}}\left[\left(\mu-\mu^{*}\right) \bar{p}+i\left(\mu+\mu^{*}\right) \bar{x}\right)\right] f(\bar{x}) \\
& \left.=\exp \left[\frac{i}{\sqrt{2}}\left(\mu+\mu^{*}\right) \bar{x}\right)\right] f\left(\bar{x}+\frac{i}{\sqrt{2}}\left(\mu^{*}-\mu\right)\right) .
\end{aligned}
$$

We can now rewrite the expression for the amplitude (97) in the following way:

$$
\begin{aligned}
& A_{n \rightarrow l}=\frac{\exp \left[-\frac{i}{\hbar} \int_{0}^{t} \gamma\left(t^{\prime}\right) d t^{\prime}+\frac{1}{4}\left(\mu^{* 2}-\mu^{2}\right)\right]}{\sqrt{\pi 2^{l+n} l ! n !}} \\
& \times \int d y \mathrm{e}^{-y^{2}-\frac{1}{2} \mu \mu^{*}} H_{l}\left(y+\frac{i}{\sqrt{2}} \mu\right) H_{n}\left(y+\frac{i}{\sqrt{2}} \mu^{*}\right) .
\end{aligned}
$$

We shall use the integral

$$
\int_{-\infty}^{+\infty} d y \mathrm{e}^{-y^{2}} H_{l}(y+w) H_{n}(y+z)=2^{n} \sqrt{\pi} z^{n-l} Q_{l}^{n-l}(-2 w z),
$$

where $l \leq n$, and $Q_{l}^{n-l}(-2 w z)$ is the Laguerre polynomial. We take $w=\frac{i}{\sqrt{2}} \mu, z=\frac{i}{\sqrt{2}} \mu^{*}$. Then we obtain for the amplitude 


$$
\begin{aligned}
& A_{n \rightarrow l}=\frac{\exp \left[-\frac{i}{\hbar} \int_{0}^{t} \gamma\left(t^{\prime}\right) d t^{\prime}+\frac{1}{4}\left(\mu^{* 2}-\mu^{2}\right)\right]}{\sqrt{\pi 2^{l+n} l ! n !}} \\
& \times \mathrm{e}^{-\frac{1}{2} \mu \mu^{*}} 2^{n} \sqrt{\pi}\left(\frac{i}{\sqrt{2}} \mu^{*}\right)^{n-l} Q_{l}^{n-l}\left(\mu \mu^{*}\right) \\
& =\frac{\exp \left[-\frac{i}{\hbar} \int_{0}^{t} \gamma\left(t^{\prime}\right) d t^{\prime}+\frac{1}{4}\left(\mu^{* 2}-\mu^{2}\right)-\frac{1}{2} \mu \mu^{*}\right]}{\sqrt{l ! n !}}\left(i \mu^{*}\right)^{n-l} \\
& \times Q_{l}^{n-l}\left(\mu \mu^{*}\right) .
\end{aligned}
$$

Since

$$
\begin{aligned}
& \left(i \mu^{*}\right)^{n-l}=\left(i|\mu| \mathrm{e}^{i \delta}\right)^{n-l}=\left(\mu \mu^{*}\right)^{\frac{n-l}{2}}\left(i \mathrm{e}^{i \delta}\right)^{n-l} \\
& =\left(\mu \mu^{*}\right)^{\frac{n-l}{2}} \mathrm{e}^{(i \omega t+i \pi / 2)(n-l)}
\end{aligned}
$$

$(\delta=\omega t)$, we finally arrive at the following expression for the amplitude $(\hbar=1)$ :

$$
A_{n \rightarrow l}=\mathrm{e}^{i \varphi(t)} \frac{1}{\sqrt{l ! n !}} \mathrm{e}^{-\frac{\rho}{2}} \rho^{(n-l) / 2} Q_{l}^{n-l}(\rho)=\mathrm{e}^{i \varphi(t)} I_{n l}(\rho),
$$

where $\rho=\mu^{*} \mu$ and

$$
\left.\varphi(t)=-\left(\int_{0}^{t} \gamma\left(t^{\prime}\right) d t^{\prime}-(\omega t+\pi / 2)(n-l)\right)+\frac{\operatorname{Im} \mu^{2}}{2}\right) .
$$

In the above Equation (102)

$$
I_{n l}(\rho)=\frac{1}{\sqrt{l ! n !}} \mathrm{e}^{-\frac{\rho}{2}} \rho^{(n-l) / 2} Q_{l}^{n-l}(\rho)
$$

is the Laguerre function (see, e.g., [2]). The distribution of probability of finding an electron at the levels $l$ is as follows:

$$
W_{l}= \begin{cases}I_{n l}^{2}(\rho), & n>l \\ I_{l n}^{2}(\rho), & l \geq n\end{cases}
$$

We know that [30]

$$
\sum_{l}(l-n) I_{n l}^{2}(\rho)=\rho, \sum_{l} I_{n l}^{2}(\rho)=1,
$$

hence for the total probability we have

$$
P=\sum_{l} W_{l}=\sum_{l} I_{n l}^{2}(\rho)=1,
$$

and

$$
\sum_{l} l I_{n l}^{2}(\rho)=n+\rho
$$

and hence the average energy in the final states $l$ (similar mathematical expression for another problem can be found in [33] Section 3, Problem 19)

$$
<E>=\omega_{c}<l+1 / 2>=E_{n}+\omega_{c} \rho .
$$


We now consider the case of a weak electromagnetic wave with $|\mu(t)| \ll 1$, when $\rho \ll 1$. Then we have the following limiting expression for the Laguerre function:

$$
I_{n l}(\rho) \rightarrow \sqrt{\frac{n !}{l !}} \frac{1}{(n-l) !} \rho^{(n-l) / 2} .
$$

Let $n-l=1$, then

$$
\begin{aligned}
& I_{n, n-1}(\rho) \rightarrow \sqrt{n} \rho^{1 / 2}, \\
& \rho=\mu^{*} \mu=\frac{|\alpha|^{2}}{\left(\omega-\omega_{c}\right)^{2}}=\frac{1}{2}\left(\frac{e_{0} b}{m}\right)^{2} \frac{e_{0} B}{\left(\omega-\omega_{c}\right)^{2}} \ll 1 .
\end{aligned}
$$

The probability of transition $n \rightarrow n-1$

$$
W_{n-1}=I_{n, n-1}^{2}=\frac{1}{2}\left(\frac{e_{0} b}{m}\right)^{2} \frac{e_{0} B n}{\left(\omega-\omega_{c}\right)^{2}}
$$

\subsection{Interband Transitions}

Let the external electromagnetic field of the electromagnetic wave be linearly polarized. The vector potentials of the fields are as follows

$$
\mathbf{A}(B)=(0, x B, 0), \mathbf{A}(t)=b(0, \sin (\vec{k} \vec{r}-\omega t), 0),
$$

where

$$
b=\left(E_{0} / \omega\right), \vec{k}=\omega(\sin \theta, 0, \cos \theta),
$$

then we have the Schrödinger equation

$$
i \partial_{t} \Psi=\left(\frac{p_{z}^{2}}{2 m}+\hat{H}\right) \Psi,
$$

where

$$
\hat{H}=\frac{1}{2 m}\left\{-\partial_{x}^{2}+\left[\hat{p}_{y}+e_{0} B x-\beta \sin (\mathbf{k r}-\omega t)\right]^{2}\right\}, \beta=e_{0} b .
$$

Here we may put $\hat{p}_{y} \rightarrow p_{y}=$ const, $\hat{p}_{z} \rightarrow p_{z}=$ const, and introduce

$$
x_{0}=-\frac{p_{y}}{e_{0} B} .
$$

The Hamiltonian operator (108) depends on $t$ and the Schrödinger equation does not have stationary solutions. Now we introduce Heisenberg operators $\hat{a}(t)$ and $\hat{a}^{+}(t)$ related to operators (81)

$$
\begin{aligned}
\hat{a}(t) & =\hat{a}-i \frac{\beta}{\sqrt{2 e_{0} B}} \sin (\mathbf{k r}-\omega t)=\hat{a}-i \frac{\eta}{\sqrt{2}} \sin (\mathbf{k r}-\omega t), \\
\eta & =\frac{\beta}{\sqrt{e_{0} B}}
\end{aligned}
$$

so that

$$
\left[\hat{a}(t), \hat{a}^{+}(t)\right]=1+\eta \cos (\mathbf{k r}-\omega t), \eta=\frac{\beta \omega}{m \omega_{c}} \sin \theta,
$$

then the Hamiltonian (108) takes the form

$$
\hat{H}=\frac{\omega_{c}}{2}\left(\hat{a}^{+}(t) \hat{a}(t)+\hat{a}(t) \hat{a}^{+}(t)\right) .
$$


The Heisenberg equation

$$
\frac{d \hat{a}(t)}{d t}=\frac{\partial \hat{a}(t)}{\partial t}+i[\hat{H}, \hat{a}(t)]
$$

proves to be inhomogeneous and nonlinear

$$
\begin{aligned}
& \frac{d \hat{a}(t)}{d t}=i \frac{\beta \omega}{\sqrt{2 e_{0} B}} \cos (\mathbf{k r}-\omega t) \\
& \quad-i \omega_{c}\left[\hat{a}(t), \hat{a}^{+}(t)\right] \hat{a}(t)-i \frac{\omega_{c}}{2}\left[\hat{a}(t)\left[\hat{a}(t), \hat{a}^{+}(t)\right]\right],
\end{aligned}
$$

which for the free harmonic oscillator goes over to the equation

$$
\frac{d \hat{a}}{d t}=-i \omega_{c} \hat{a}
$$

It should be noted that (111) is an exact equation, taking into account interaction of an electron with the magnetic field and the electromagnetic wave. Its nonlinearity may be neglected if the last term in (111) is small

$$
\frac{\omega_{c} \sqrt{2 e_{0} B} \eta}{\beta \omega} \sim \frac{\omega_{c} \sqrt{2 e_{0} B} \beta \omega}{\beta \omega m \omega_{c}}=\frac{\sqrt{2 e_{0} B}}{m}=\sqrt{\frac{2 \omega_{c}}{m}} \ll 1,
$$

which is evidently true for any laboratory magnetic fields $B \ll B_{0}$, where $B_{0}=m^{2} / e_{0}=4,4 \times 10^{13} G$. Then

$$
\left[\hat{a}(t)\left[\hat{a}(t), \hat{a}^{+}(t)\right]\right] \approx 0,
$$

and $\cos (\vec{k} \vec{r}-\omega t) \approx \cos \left(\phi_{0}-\omega t\right), \phi_{0}=$ const. As a result, we obtain the equation for parametric oscillator

$$
\frac{d \hat{a}(t)}{d t}=i \frac{\beta \omega}{\sqrt{2 e_{0} B}} \cos \left(\phi_{0}-\omega t\right)-i \omega(t) \hat{a}(t),
$$

where

$$
\omega(t)=\omega_{\mathcal{c}}\left(1+\eta \cos \left(\phi_{0}-\omega t\right)\right) .
$$

One should note that once again

$$
\frac{\omega_{c} \eta}{\beta \omega / \sqrt{2 e B}}=\frac{\sqrt{2 e_{0} B}}{m} \ll 1,
$$

so that we can put $\omega(t) \approx \omega_{c}$.

Now, we may write the solutions of (113) in two ways

$$
\begin{aligned}
& \hat{a}(t)=\tilde{a}_{\text {in }}(t)+\int_{-\infty}^{t} d t^{\prime} \chi\left(t^{\prime}\right) \exp \left[i \int_{t^{\prime}}^{t} \omega(\tau) d \tau\right], \\
& \hat{a}(t)=\tilde{a}_{\text {out }}(t)-\int_{t}^{\infty} d t^{\prime} \chi\left(t^{\prime}\right) \exp \left[i \int_{t^{\prime}}^{t} \omega(\tau) d \tau\right] .
\end{aligned}
$$


In these formulas we introduced the following operators:

$$
\begin{aligned}
\tilde{a}_{\text {in }}(t) & =\hat{a}_{\text {in }} \exp \left[i \omega_{c} t+i \frac{e_{0} b}{m} \sin \left(\omega t-\phi_{0}\right)\right], \\
\tilde{a}_{\text {out }}(t) & =\hat{a}_{\text {out }} \exp \left[i \omega_{c} t+i \frac{e_{0} b}{m} \sin \left(\omega t-\phi_{0}\right)\right] \\
\chi(t) & =i \frac{\beta \omega}{\sqrt{2 e_{0} B}} \cos \left(\omega t-\phi_{0}\right)
\end{aligned}
$$

where $\hat{a}_{i n}, \hat{a}_{\text {out }}$ are some yet undefined constant operators. Let potential $\vec{A}(t)$ is switched on adiabatically at $t \rightarrow-\infty$ and is switched off at $t \rightarrow \infty$

$$
b \rightarrow 0, \text { at } t \rightarrow \pm \infty, \frac{d b / d t}{b} \ll \omega_{c} .
$$

Under the influence of external magnetic and wave fields in the infinite time interval $(-\infty,+\infty)$ the operator $\hat{a}_{\text {in }}$ goes over to $\hat{a}_{\text {out }}=\hat{a}_{\text {in }}+\tilde{\mu}$, where

$$
\tilde{\mu}=\int_{-\infty}^{+\infty} d t^{\prime} \chi\left(t^{\prime}\right) \exp \left[-i \omega_{c} t^{\prime}+i \frac{e_{0} b}{m} \sin \left(\omega t^{\prime}-\phi_{0}\right)\right] .
$$

Relation of operators $\hat{a}_{\text {out }}$ and $\hat{a}_{\text {in }}$ can be written down with the help of the so-called $\hat{s}$-matrix

$$
\hat{a}_{\text {out }}=\hat{S}^{+} \hat{a}_{i n} \hat{S}, \hat{s}=\exp \left[\tilde{\mu} \hat{a}_{i n}^{+}-\tilde{\mu}^{*} \hat{a}_{i n}\right], \hat{S}^{+} \hat{S}=I .
$$

With the use of this matrix one may find an exact analytic expression for the probability of transition of an electron under the influence of a magnetic field and a strong electromagnetic wave field.

Moreover, if we consider the transition from the valence band to the conduction band under the influence of the alternating field $\mathbf{E}=\mathbf{e} E_{0} \exp \left(-i \omega^{\prime} t\right)$, then in such a transition, the selection rules are satisfied for the wave numbers $k_{z 1}=k_{z 2}, k_{y 1}=k_{y 2}$, but not for the quantum numbers $n^{\prime}$, since transitions to the states $n^{\prime} \neq n$ are possible when $\eta \neq 0$. Then the optical absorption coefficient can be found, which is an exact expression in the magnetic field $B$ and wave field $F=b \omega$ (see [31])

$$
\begin{aligned}
& \alpha(F, B)=\frac{\left(\mathbf{p}_{12} \mathbf{e}\right)^{2} e^{3} B\left(2 m^{*}\right)^{1 / 2}}{n_{0} m \omega^{\prime}} \sum_{n^{\prime}, n} I_{n^{\prime} n}^{2}\left(\eta_{1,2}\right) \\
& \times\left[\omega^{\prime}+\left(n^{\prime}-n\right) \omega-\omega_{C 1}(n+1 / 2)-\omega_{C 2}\left(n^{\prime}+1 / 2\right)-\epsilon_{g}\right. \\
& \left.-\frac{e^{2} F^{2}}{2 m^{*}\left(\omega+\omega_{C 1}\right)\left(\omega-\omega_{C 2}\right)}\right]^{-1 / 2} .
\end{aligned}
$$

where $\mathbf{p}_{12}$ is the matrix element of the dipole transition at $k=0$, and the dimensionless parameter is introduced

$$
\eta_{1,2}=\frac{e^{2} F^{2}}{\left[m^{*} r_{0}\left(\omega+\omega_{C 1}\right)\left(\omega-\omega_{C 2}\right)\right]} .
$$

Here $\omega_{C 1}$ and $\omega_{C 2}$ are the cyclotron frequencies in bands 1 and 2, and $m^{*}$ is the reduced mass. The maximum absorption will be observed for resonant values of the frequency $\omega^{\prime}$, i.e., for zero values of the expression in square brackets in the above expression, but with this $\alpha$ will nevertheless be finite due to the presence of damping. When $n^{\prime}-n$ is large, the function $I_{n^{\prime} n}$ behaves like the Bessel function $J_{n^{\prime}-n}\left(2 \sqrt{n \eta_{1,2}}\right)$, leading to oscillation of $\alpha$ depending on the value of $\eta_{1,2}$. For strong field with $\eta_{1,2} \gg 1$, for transitions between small quantum numbers, the matrix element proportional to $\mathrm{e}^{-\eta_{1,2} / 2}$ will yield an exponential damping of the transition probability.

When $\omega \ll \omega_{C}$ the electron moves in an almost constant field and the result (120) goes over into the corresponding formula obtained by Aronov [34] for constant crossed fields. The parameter $\eta_{1,2}$ for 
intense microwave or infrared radiation can become close to unity $\left(\eta_{1,2} \sim 1\right)$. To this end it is necessary to specify, for example, the values $m^{*}=10^{-28} \mathrm{~g}$, $\omega=10^{13} \mathrm{sec}, B=10^{4} \mathrm{G}$, and an electric field intensity $F=10^{4} \mathrm{~V} / \mathrm{cm}$, which are typical values for experimental conditions.

\section{Klein-Gordon Equation}

To begin with, we can try to solve a problem for a "scalar" particle described by the squared Dirac equation without the spin terms in the space-time with dimensions $\mathrm{D}=3+1$ temporarily with a mass term $m^{2}$, i.e., the Klein-Gordon equation

$$
-\partial_{t}^{2} \Psi=\left(-\partial_{z}^{2}+P_{x}^{2}+P_{y}^{2}+m^{2}\right) \Psi,
$$

which gives

$$
\begin{aligned}
-\left(\partial_{t}^{2}-\partial_{z}^{2}\right) \Psi & =\left\{-\left[\partial_{x}+i \beta \cos \omega(t-z)\right]^{2}\right. \\
& \left.-\left[\partial_{y}+i e_{0} B x+i \beta \sin \omega(t-z)\right]^{2}+m^{2}\right\} \Psi .
\end{aligned}
$$

Let us introduce new variables

$$
\tau=t-z, \eta=\frac{1}{2}(t+z)
$$

then $\partial_{t}^{2}-\partial_{z}^{2}=-2\left(-i \partial_{\tau}\right)\left(-i \partial_{\eta}\right)$.

We will search for a solution

$$
\Psi=\mathrm{e}^{-i p_{\tau} \tau} \mathrm{e}^{i p_{\eta} \eta} \mathrm{e}^{i p_{y} y} \psi(\tau, x),
$$

where we considered that there is no dependence on $\eta$ and $y$ in the equation, and hence the momenta $\hat{p}_{\eta}=-i \partial_{\eta} \rightarrow p_{\eta}=$ const and $\hat{p}_{y}=-i \partial_{y} \rightarrow p_{y}=$ const are conserved, and $p_{\tau}$ is a new still undefined quantum number. Thus,

$$
\begin{aligned}
& -\left(\partial_{t}^{2}-\partial_{z}^{2}\right) \Psi=-2\left(-i \partial_{\tau}\right)\left(-i \partial_{\eta}\right) \Psi \\
& =2 p_{\eta} \mathrm{e}^{-i p_{\tau} \tau} \mathrm{e}^{i p_{\eta} \eta} \mathrm{e}^{i p_{y} y}\left[\left(i \partial_{\tau}\right)+p_{\tau}\right] \psi(\tau, x)
\end{aligned}
$$

Equation (121) takes the form

$$
\begin{aligned}
& 2 p_{\eta}\left[\left(i \partial_{\tau}\right)+p_{\tau}\right] \psi(\tau, x)=\left\{-\left[\partial_{x}+i \beta \cos \omega \tau\right]^{2}\right. \\
& \left.-\left[\partial_{y}+i e_{0} B x+i \beta \sin \omega \tau\right]^{2}+m^{2}\right\} \psi(\tau, x) .
\end{aligned}
$$

The quantum number $p_{\tau}$ may now be related to the other quantum number $p_{\eta}$, if we put $2 p_{\tau} p_{\eta}=m^{2}$. Let $m^{2} \rightarrow 0$. One can see that the obtained equation has the form of a certain Schrödinger equation for a particle of mass $p_{\eta}$ in an external $\tau$-dependent electromagnetic wave field. Now $2 i \partial_{\tau}=i\left(\partial_{t}+\partial_{z}\right)$, and $i \partial_{\eta}=-i\left(\partial_{t}-\partial_{z}\right)$, so that we may rename $p_{\tau}=\frac{1}{2}\left(p_{0}-p_{z}\right)$ and $p_{\eta}=\left(p_{0}+p_{z}\right)$, and $2 p_{\tau} p_{\eta}=m^{2} \rightarrow 0$ means $\left(p_{0}^{2}-p_{z}^{2}\right)=\left(p_{0}-p_{z}\right)\left(p_{0}+p_{z}\right) \rightarrow 0$, and we may put $p_{\tau}=\frac{1}{2}\left(p_{0}-p_{z}\right)=0, p_{0}+p_{z}=p_{\eta}, p_{\eta}=2 p_{0}$. We arrive at the following equation:

$$
\begin{aligned}
& i \partial_{\tau} \psi(\tau, x)=\frac{1}{2 p_{\eta}}\left\{\left[\hat{p}_{x}+\beta \cos \omega \tau\right]^{2}\right. \\
& \left.+\left[p_{y}+e_{0} B x+\beta \sin \omega \tau\right]^{2}\right\} \psi(\tau, x) .
\end{aligned}
$$


It is evident that the above Equation (125) for the wave function (122) is immediately related to Equations (77) and (78) with the solution (94)-(96), if the following replacements are made:

$$
t \rightarrow \tau, m \rightarrow p_{\eta}, \omega_{c} \rightarrow \Omega=\frac{e_{0} B}{p_{\eta}} .
$$

\subsection{Pure Magnetic Field}

Let us try to prove this result in a pure magnetic field. We have in $\mathrm{D}=3+1$ :

$$
\left(-\partial_{t}^{2}+\partial_{z}^{2}\right) \Psi=\left\{-\partial_{x}^{2}-\left(\partial_{y}+i e_{0} B x\right)^{2}\right\} \Psi .
$$

Let us introduce new variables

$$
\tau=t-z, \eta=\frac{1}{2}(t+z),
$$

then $\partial_{t}^{2}-\partial_{z}^{2}=-2\left(-i \partial_{\tau}\right)\left(-i \partial_{\eta}\right)$. We will search for a solution

$$
\Psi=\mathrm{e}^{-i p_{\tau} \tau} \mathrm{e}^{i p_{\eta} \eta} \mathrm{e}^{i p_{y} y} \psi(\tau, x),
$$

Thus,

$$
\begin{aligned}
& -\left(\partial_{t}^{2}-\partial_{z}^{2}\right) \Psi=-2\left(-i \partial_{\tau}\right)\left(-i \partial_{\eta}\right) \Psi \\
& =2 p_{\eta} \mathrm{e}^{-i p_{\tau} \tau} \mathrm{e}^{i p_{\eta} \eta} \mathrm{e}^{i p_{y} y}\left[\left(i \partial_{\tau}\right)+p_{\tau}\right] \psi(\tau, x)
\end{aligned}
$$

Equation (121) in pure magnetic field with $\eta=0$ takes the form

$$
\begin{aligned}
& 2 p_{\eta}\left[\left(i \partial_{\tau}\right)+p_{\tau}\right] \psi(\tau, x) \\
& =\left\{-\partial_{x}^{2}-\left(\partial_{y}+i e_{0} B x\right)^{2}+m^{2}\right\} \psi(\tau, x) .
\end{aligned}
$$

The quantum number $p_{\tau}$ may now be related to the other quantum number $p_{\eta}$, if we put $2 p_{\tau} p_{\eta}=m^{2}$. Let $m^{2} \rightarrow 0$, then $p_{\tau}=0$. One can see that the obtained equation has the form of a certain Schrödinger equation for a particle of mass $p_{\eta}$ in an external magnetic field

$$
i \partial_{\tau} \psi(\tau, x)=\frac{1}{2 p_{\eta}}\left\{\left[\hat{p}_{x}\right]^{2}+\left[p_{y}+e_{0} B x\right]^{2}\right\} \psi(\tau, x) .
$$

The operator in the right-hand side of the above equation has eigenvalues

$$
\frac{1}{2 p_{\eta}}\left\{\hat{p}_{x}^{2}+\left(e_{0} B\right)^{2}\left(x-x_{0}\right)^{2}\right\} \rightarrow \Omega\left(n+\frac{1}{2}\right)
$$

then

$$
i \partial_{\tau} \psi(\tau, x)=\Omega\left(n+\frac{1}{2}\right) \psi(\tau, x), \Omega=\frac{e_{0} B}{p_{\eta}} .
$$

We search for a "stationary" solution $\psi(\tau, x) \sim \mathrm{e}^{-i \epsilon \tau}$, then $i \partial_{\tau} \psi(\tau, x)=\epsilon \psi(\tau, x)$ and

$$
\epsilon \psi(\tau, x)=\frac{e_{0} B}{p_{\eta}}\left(n+\frac{1}{2}\right) \psi(\tau, x)
$$


To get the familiar result for $\mathrm{D}=3+1$, we must put

$$
\epsilon p_{\eta}=\frac{\varepsilon^{2}-p_{z}^{2}}{2}
$$

so that

$$
\frac{\varepsilon^{2}-p_{z}^{2}}{2 p_{\eta}}=e_{0} B\left(n+\frac{1}{2}\right)
$$

In the non-relativistic limit $p_{\eta} \rightarrow m$ with $p_{z}=0$ we have

$$
\epsilon=\frac{e_{0} B\left(n+\frac{1}{2}\right)}{m}
$$

which is the well-known result.

\subsection{Superposition}

With the help of the result (94) and (95), (96) we now arrive at the following solutionfor the Klein-Gordon equation in the superposition of a magnetic and a plane circularly polarized electromagnetic field in the case of a vanishing $m \rightarrow 0$, when $p_{\tau}=0$

$$
\begin{aligned}
& \Psi_{p_{y}, p_{\eta}}=\mathrm{e}^{i p_{y} y} \mathrm{e}^{i p_{\eta} \eta} \mathrm{e}^{-i \epsilon \tau} \Lambda u_{n}(\bar{x}) \\
& =\mathrm{e}^{i p_{y} y} \mathrm{e}^{i p_{\eta} \eta} \mathrm{e}^{-i \epsilon \tau} \mathrm{D}_{\mu} u_{n}(\bar{x}) \exp \left(-i \int_{0}^{\tau} \gamma(\tau) d \tau\right) \\
& =\mathrm{e}^{i p_{y} y} \mathrm{e}^{i p_{\eta} \eta} \bar{\Phi}(\tau, x) .
\end{aligned}
$$

Following the non-relativistic solution (85),

$$
\chi=\mathrm{D}_{\mu}^{-1} \bar{\Phi} \exp \left(i \int_{0}^{\tau} \gamma(\tau) d \tau\right)
$$

we define the function $\chi(\tau, x) \sim \mathrm{e}^{-i \epsilon \tau}$ that satisfies the equation

$$
i \partial_{\tau} \chi(\tau, x)=\frac{1}{2 p_{\eta}}\left\{\hat{p}_{x}^{2}+\left(e_{0} B\right)^{2}\left(x-x_{0}\right)^{2}\right\} \chi(\tau, x),
$$

which is just Equation (133), and hence

$$
i \partial_{\tau} \chi(\tau, x)=\Omega\left(n+\frac{1}{2}\right) \chi(\tau, x), \Omega=\frac{e_{0} B}{p_{\eta}}
$$

and we have

$$
\epsilon_{n}=\frac{\varepsilon_{n}^{2}}{2 p_{\eta}}, \quad \varepsilon_{n}^{2}=2 e_{0} B\left(n+\frac{1}{2}\right) .
$$

For $n \gg 1$ this gives

$$
\epsilon_{n}-\epsilon_{n-1}=\frac{\varepsilon_{n}^{2}-\varepsilon_{n-1}^{2}}{2 p_{\eta}}=\frac{e_{0} B}{p_{\eta}}=\Omega .
$$

Another proof of the above result let us consider the problem in the magnetic field alone putting electromagnetic wave field vanishing: $b \rightarrow 0$, or $\eta \rightarrow 0$. We consider the Klein-Gordon Equation (121) as it is for $\mathrm{D}=2+1\left(p_{z}=0, \eta=0, m^{2}=0\right)$

$$
-\partial_{t}^{2} \Psi=\left\{-\partial_{x}^{2}-\left(\partial_{y}+i e_{0} B x\right)^{2}\right\} \Psi .
$$


The operator in the right-hand side of the above equation has eigenvalues

$$
-\partial_{x}^{2}-\left(\partial_{y}+i e_{0} B x\right)^{2} \rightarrow 2 e B\left(n+\frac{1}{2}\right)
$$

and for the stationary solutions $\Psi \sim \mathrm{e}^{-i p_{0} t}$ we have

$$
p_{0}^{2}=\varepsilon_{n}^{2}=2 e_{0} B\left(n+\frac{1}{2}\right),
$$

which is the well-known result.

\subsection{Limit $m \rightarrow 0, p_{z} \rightarrow \infty$}

The Klein-Gordon equation

$$
-\partial_{t}^{2} \Psi=\left(-\partial_{z}^{2}+P_{x}^{2}+P_{y}^{2}+m^{2}\right) \Psi,
$$

can be written as

$$
\begin{aligned}
\partial_{t}^{2} \Psi & =\left\{\left[\partial_{x}+i \beta \cos \omega(t-z)\right]^{2}\right. \\
& \left.+\left[\partial_{y}+i e B x+i \beta \sin \omega(t-z)\right]^{2}-p_{z}^{2}-m^{2}\right\} \Psi .
\end{aligned}
$$

If we put $m \rightarrow 0$, we have

$$
\begin{aligned}
\partial_{t}^{2} \Psi & =\left\{\left[\partial_{x}+i \beta \cos \omega(t-z)\right]^{2}\right. \\
& \left.\left.+\left[\partial_{y}+i e_{0} B x+i \beta \sin \omega(t-z)\right)\right]^{2}-p_{z}^{2}\right\} \Psi .
\end{aligned}
$$

We write $\Psi=\mathrm{e}^{-i p_{z} t} \Phi$ and we quantize $p_{z}$ momentum with periodic boundary conditions in the third direction

$$
p_{z}=\frac{2 \pi n}{L_{z}}, n=0, \pm 1, \pm 2, \ldots
$$

For very thin slab in the $z$-direction, $L_{z} \rightarrow 0$, we have $z \rightarrow 0, p_{z} \rightarrow \infty$. This supplies a restriction on the maximum extension $L_{z}$ along the $z$-axis and the minimum value of $p_{z}$

$$
L_{z} \leq L_{z}^{\max }=\frac{2 \pi}{\sqrt{e_{0} B}}, \quad p_{z} \geq p_{z}^{\min }=\frac{\sqrt{e_{0} B}}{2 \pi} .
$$

Then we have

$$
\begin{aligned}
i \partial_{t} \Phi & =\frac{1}{2 p_{z}}\left\{\left[-i \partial_{x}+\beta \cos \omega t\right]^{2}\right. \\
& \left.\left.+\left[-i \partial_{y}+e B x+\beta \sin \omega t\right)\right]^{2}\right\} \Phi .
\end{aligned}
$$

which is just Equation (125) (or Equation (137) for $\eta=0$ ).

\section{Electron in a Quantum Well in the Conduction Band}

In recent years, studies of the optical absorption in quantum wells have been made under the influence of various external factors. Let us assume now that an electron is confined in a quantum well in the conduction band and it is not only under the influence of an external magnetic field but also of an electromagnetic wave. Then its free motion along the $z$-axis described by plane-wave solution 
of Equation (72) $\exp \left(i p_{z} z\right)$ should be replaced by a solution of the Schrödinger equation with the confining potential

$$
i \partial_{t} \Phi=\frac{1}{2 m}\left(-\partial_{z}^{2}+P_{x}^{2}+P_{y}^{2}\right) \Phi+V(z) \Phi,
$$

The form of the confining potential $V(z)$ was earlier proposed (see, e.g., [21-24]) in the form (we here retain physical unit for Planck constant $\hbar$ )

$$
V(z)= \begin{cases}\frac{1}{2} m \omega_{z}^{2} z^{2}+\frac{\hbar^{2} q}{2 m z^{2}}, & z \geq 0 \\ \infty, & z<0\end{cases}
$$

Here the following notations were used: $\omega_{z}$ is the confinement frequency and $q$ is the characteristic parameter with the inverse squared potential. Since separation of variables can be realized, the motion in the $z$-direction is described by the equation for $\phi(z)$ for $z \geq 0$

$$
\mathcal{E} \phi(z)=\left[-\frac{\hbar^{2}}{2 m} \partial_{z}^{2}+\frac{1}{2} m \omega_{z}^{2} z^{2}+\frac{\hbar^{2} q}{2 m z^{2}}\right] \phi(z) .
$$

Defining the new dimensionless variable $\zeta$ and energy parameter $\lambda$

$$
\zeta=\left(\frac{z}{\alpha_{z}}\right)^{2}, \text { where } \alpha_{z}=\left(\frac{\hbar}{m \omega_{z}}\right)^{1 / 2}, \lambda=\frac{\mathcal{E}}{\hbar \omega_{z}},
$$

the above equation can be rewritten as

$$
\frac{d^{2} \phi}{d \zeta^{2}}+\frac{1}{2 \zeta} \frac{d \phi}{d \zeta}-\left[\frac{1}{4}+\frac{q}{4 \zeta^{2}}-\frac{\lambda}{2 \zeta}\right] \phi=0
$$

The solution of this type of equations in terms of associated Laguerre polynomials is well known in quantum mechanics in relation, e.g., with the problem of synchrotron radiation or hydrogen atom and can be found, e.g., in [2,30]. The particular solution of Equation (145) looks like

$$
\phi_{s}(z)=N_{s} z^{2 \sigma} \mathrm{e}^{-\zeta / 2} L_{S}^{\delta}(\zeta),
$$

where $s=0,1,2, \ldots, N_{s}=\sqrt{2 s ! / \Gamma(2 \sigma+s+1 / 2)}, \sigma=(\sqrt{1 / 4+q}+1 / 2) / 2$, and $L_{s}^{\delta}(\zeta)$ is the associated Laguerre polynomial, $\delta=2 \sigma-1 / 2$. The energy spectrum of $z$-oscillations corresponding to this solution follows [23]

$$
\mathcal{E}_{s}=\omega_{z}(2 s+1+\sqrt{q+1 / 4})
$$

To make an exact account for the electromagnetic wave also, we are able now to combine the already found above (Section 3) part of the wave function responsible for electron motion in $x O y$-plane (94)-(96) with this solution (146), (147) that describes the confined motion along $z$-axis, yielding

$$
\Phi_{p_{y}, p_{z}, n}=\mathrm{e}^{i p_{y} y} \mathrm{e}^{-i \varepsilon_{n, s} t} \mathrm{D}_{\mu} u_{n}(\bar{x}) \mathrm{e}^{\left(-i \int_{0}^{t} \gamma(t) d t\right)} \phi_{s}(z),
$$

with the energy

$$
\begin{aligned}
& \varepsilon_{n, s}=\varepsilon_{n}+\mathcal{E}_{s} \\
& =\omega_{c}(n+1 / 2)+\omega_{z}(2 s+1+\sqrt{q+1 / 4}) .
\end{aligned}
$$

It was demonstrated, for instance, in [21-23], that due to inclusion of the confining potential, the optical absorption coefficients are strongly affected not only by a static magnetic field, but also by 
the confinement frequency and the incident optical intensity, which in our solution is exactly taken into account.

\section{Conclusions}

In this paper, we considered motion of a massless quasi-particle in graphene and demonstrated that its motion is principally different from motion of a massive particle in an external field. The revolution frequency of a quasi-particle (electron) in a magnetic field ("cyclotron frequency") depends on its energy. In solving the equation of motion of an electron in the resonance condition, i.e., when the wave frequency coincides with the "cyclotron frequency", in the case of a weak wave, it is shown that equations for the deviation of the phase of motion from its constant value is reduced to the equation of nonlinear oscillations. In a special case, when the wave frequency is twice that of the "cyclotron frequency" an exact solution for the equation of motion of an electron in the wave of arbitrary strength was obtained. This solution can be interpreted from the point of view of the solution of the equation of motion of an electron as wave frequency-halving. For a relativistic electron, in the case when its spin states can be neglected, the Klein-Gordon equation in a superposition of a plane electromagnetic wave and a constant magnetic field was studied. Its relation to non-relativistic equation and, on the contrary, to the massless limit $m \rightarrow 0$ equation was discussed. Moreover, we studied electron radiative transitions in semiconductor in two cases: intrabank transitions and interband transitions. We also considered electron in a quantum well in the conduction band, i.e., when an electron is not only under combined influence of an external magnetic field and electromagnetic wave, but also is confined in a quantum well in the conduction band. We were able to combine the already found part of the wave function responsible for electron motion in $x O y$-plane with the solution that describes the confined motion along $z$-axis (148), and hence, according to the results in [21-23], due to inclusion of the confining potential, the optical absorption coefficients are strongly affected not only by a static magnetic field, but also by the confinement frequency and the incident optical intensity. It is, therefore, quite reasonable that corresponding calculations that would generalize our above estimates of the absorption coefficient (120), be realized with the above wave function, with the incident optical intensity exactly taken into account in our solution. We are planning to do this in our forthcoming publications.

Funding: This research received no external funding.

Acknowledgments: The author would like to thank A.V. Borisov, A.E. Lobanov and E.S. Durandina for valuable discussions.

Conflicts of Interest: The author declares no conflicts of interest.

\section{References}

1. Sokolov, A.A.; Ternov, I.M. Radiation from Relativistic Electrons; Kilmister, C.W., Ed.; Translation Series; American Institute of Physics: College Park, MD, New York. 1986.

2. Sokolov, A.A.; Ternov, I.M. Sokolov, A. A., Ternov, I. M. Synchrotron Radiaton; Akademie: Berlin, Germany, Pergamon Press: New York, NY, USA, 1968.

3. Bordovitsyn, V.A. (Ed.) Synchrotron Radiation Theory and Its Development: In Memory of I. M. Ternov; World Scientific Pub. Co. Pte. Ltd.: Singapore, 1999.

4. Sokolov, A.; Zhukovskii, V.; Nikitina, N. Quantum transitions of relativistic electrons in a superstrong magnetic field. Phys. Lett. A 1973, 43, 85. [CrossRef]

5. Sokolov, A.A.; Borisov, A.V.; Zhukovskii, V.C.Synchrotron radiation from relativistic electrons in intense magnetic fields, Izvestiya Vysshikh Uchebnykh Zavedenii. Fizika 1975, 10, 51-57.

6. Sokolov, A.A.; Ternov, I.M., Polarization and spin effects in the theory of synchrotron radiation. Dokl. Akad. Nauk SSSR 1963, 153, 1052.

7. Akhmedov, E.T.; Singleton, D. On the relation between Unruh and Sokolov-Ternov effects. Int. J. Mod. Phys. A 2007, 22, 4797. [CrossRef] 
8. Abakumova E. V., Achasov M. N., Berkaev D. E., et al., Backscattering of Laser Radiation on Ultrarelativistic Electrons in a Transverse Magnetic Field: Evidence of MeV-Scale Photon Interference; Phys. Rev. Let. 2013, 110, 140402. [CrossRef] [PubMed]

9. Zhukovsky, V.C.; Herrmann, I. Compton- effect and induced Compton-effect in a constant eletromagnetic field Soviet. J. Nucl. Phys. 1971, 14, 85.

10. Morimoto, T.; Hatsugai, Y.; Aoki, H. Cyclotron radiation and emission in graphene-A possibility of landau-level laser. J. Phys. Conf. Ser. 2009, 150, 022059. [CrossRef]

11. Mikhailov, S.A. Non-linear electromagnetic response of graphene. Europhys. Lett. 2007, 79, 27002. [CrossRef]

12. Mikhailov, S.A.; Ziegler, K. Nonlinear electromagnetic response of graphene: frequency multiplication and the self-consistent-field effects. J. Phys. Condens. Matter 2008, 20, 384204. [CrossRef] [PubMed]

13. Mikhailov, S.A. Nonlinear cyclotron resonance of a massless quasiparticle in graphene. Phys. Rev. B 2009, 79, 241309(R). [CrossRef]

14. Neto, A.H.C.; Guinea, F.; Peres, N.M.R.; Novoselov, K.S.; Geim, A.K. The electronic properties of graphene. Rev. Mod. Phys. 2009, 81, 109. [CrossRef]

15. Keldysh, L.V. Ionization in the field of a strong electromagnetic wave. Sov. Phys. JETP. 1964, 47, 1945-1957.

16. Zhukovsky, K. High-harmonic x-ray undulator radiation for nanoscale-wavelength free-electron lasers. J. Phys. D Appl. Phys. 2017, 50, 505601; Corrigendum J. Phys. D Appl. Phys. 2018, 51, 149501. [CrossRef]

17. Vshivtsev, A.S.; Zhukovskii, V.C.; The polarization of radiation in an undulator, Moscow University Physics Bulletin 1975, 30, 13.

18. Zhukovsky, K.; Kalitenko, A. Phenomenological and numerical analysis of power evolution and bunching in single-pass X-ray FELs. J. Synchrotron Radiat. 2019, 26, 159. [CrossRef]

19. Stepanov, E.A.; Zhukovsky, V.C. Publisher's Note: Graphene under the influence of Aharonov-Bohm flux and constant magnetic field. Phys. Rev. B 2016, 94, 094101. [CrossRef]

20. Ebert, D.; Klimenko, K.G.; Kolmakov, P.B.; Zhukovsky, V.C.. Phase transitions in hexagonal, graphene-like lattice sheets and nanotubes under the influence of external conditions. Ann. Phys. 2016, 371, 254. [CrossRef]

21. Tung, L.V.; Vinh, P.T.; Phuc, H.V. Magneto-optical properties of semi-parabolic plus semi-inverse squared quantum wells. Phys. B Condens. Matter 2018; 539, 117. [CrossRef]

22. Nguyen, C.V.; Hieu, N.N.; Poklonski, N.A.; Ilyasov, V.V.; Dinh, L.; Phong, T.C.; Tung, L.V.; Phuc, H.V. Magneto-optical transport properties of monolayer MoS2 on polar substrates. Phys. Rev. B 2017, 96, 125411. [CrossRef]

23. Hassanabadi, H.; Liu, G.; Lu, L. Nonlinear optical rectification and the second-harmonic generation in semi-parabolic and semi-inverse squared quantum wells. Solid State Commun. 2012, 152, 1761. [CrossRef]

24. Liu, G.; Guo, K.; Wang, C. Linear and nonlinear intersubband optical absorption in a disk-shaped quantum dot with a parabolic potential plus an inverse squared potential in a static magnetic. Physica B 2012, 407, 2334. [CrossRef]

25. Zhilich, A.G.; Monozon, B.S. Multiphoton magnetoabsorption in narrow-band semiconductors in crossed fields. Zh. Eksp. Teor. Fiz. 1980, 78, 1087.

26. Rylyuk, V.M.; Ortner, J. Decay of a weakly bound level in a monochromatic electromagnetic field and a static magnetic field. Phys. Rev. A. 2003, 67, 013414. [CrossRef]

27. Vshivtsev, A.S.; Magnitsky, B.V.; Zhukovsky, V.C.; Klimenko, K.G. Dynamical effects in (2+ 1)-dimensional theories with four-fermion interaction. Phys. Part. Nucl. 1998, 29, 523. [CrossRef]

28. Hagerstrom, A.; Wu, M.; Eykholt, R.; Kalinikos, B.A. Tuning of chaotic surface spin waves in a magnetic-film feedback ring via the ring gain. Phys. Rev. B. 2011, 83, 104402. [CrossRef]

29. Wu, M.; Hagerstrom, A.M.; Eykholt, R.; Kondrashov, A.; Kalinikos, B.A. Excitation of chaotic spin waves through modulational instability. PRL 2009, 102, 237203. [CrossRef]

30. Sokolov, A.A.; Ternov, I.M.; Zhukovskii, V.C. Quantum Mechanics; MIR: Moscow, Russia, 1984.

31. Zhukovskii, V.C.; Sokolov, A.A. Interband transitions in semiconductors in a magnetic field under the influence of a strong electromagnetic wave. JETP LETTERS-USSR 1967, 9, 311.

32. Bergou, J.; Varró, S. On the quantum mechanical states of a free charged particle in a uniform magnetic field and a laser field, J. Phys. B At. Mol. Phys. 1982, 15, L179-L184. [CrossRef] 
33. Gol'dman, I.I.; Krivchenkov Problems in Quantum Mechanics; Dover Publications, Inc.: New York, NY, USA, 2006.

34. Aronov, A.G. Oscillations of the optical absorption coefficient in crossed electric and magnetic fields. Fiz. Tverd. Tela. 1963, 5, 552.

(C) 2020 by the authors. Licensee MDPI, Basel, Switzerland. This article is an open access article distributed under the terms and conditions of the Creative Commons Attribution (CC BY) license (http://creativecommons.org/licenses/by/4.0/). 